Utah State University

DigitalCommons@USU

\title{
$5-2013$
}

\section{Minimizing the Disruption of Traffic Flow of Automated Vehicles During Lane Changes}

Divya Desiraju

Utah State University

Follow this and additional works at: https://digitalcommons.usu.edu/etd

Part of the Computer Engineering Commons

\section{Recommended Citation}

Desiraju, Divya, "Minimizing the Disruption of Traffic Flow of Automated Vehicles During Lane Changes" (2013). All Graduate Theses and Dissertations. 2010.

https://digitalcommons.usu.edu/etd/2010

This Thesis is brought to you for free and open access by the Graduate Studies at DigitalCommons@USU. It has been accepted for inclusion in All Graduate Theses and Dissertations by an authorized administrator of DigitalCommons@USU. For more information, please contact digitalcommons@usu.edu.

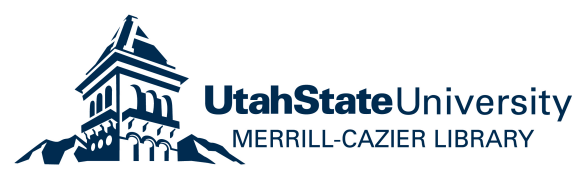




\title{
MINIMIZING THE DISRUPTION OF TRAFFIC FLOW OF AUTOMATED VEHICLES DURING LANE CHANGES
}

\author{
by \\ Divya Desiraju \\ A thesis submitted in partial fulfillment \\ of the requirements for the degree \\ of \\ MASTER OF SCIENCE \\ in \\ Computer Engineering
}

Approved:

Dr. Tam Chantem

Dr. Edmund Spencer

Major Professor

Committee Member

Dr. Chris Winstead

Dr. Mark R. McLellan

Committee Member

Vice President for Research and

Dean of the School of Graduate Studies

\section{UTAH STATE UNIVERSITY \\ Logan, Utah}


Copyright C Divya Desiraju 2013

All Rights Reserved 


\begin{abstract}
Minimizing the Disruption of Traffic Flow of Automated Vehicles During Lane Changes

by
\end{abstract}

Divya Desiraju, Master of Science

Utah State University, 2013

Major Professor: Dr. Tam Chantem

Department: Electrical and Computer Engineering

In intelligent transportation systems, most of the research work has focused on lane change assistant systems. No existing work considers minimizing the interruption of traffic flow by maximizing the number of lane changes while eliminating the collisions. In this thesis, we develop qualitative and quantitative approaches for minimizing the interruption of traffic flow for three lane scenarios and show that we can extend our approach to any random number of lanes. The algorithm we propose in this thesis is able to achieve the maximum number of lane changes provided that only one vehicle per group (novel concept which is described in this thesis) is allowed to change lanes at a time. Simulation results show that our approach provides much better performance when compared with different lane change algorithms without incurring large overhead, and is hence suitable for online use.

(39 pages) 


\section{Public Abstract}

Minimizing the Disruption of Traffic Flow of Automated Vehicles During Lane Changes

by

Divya Desiraju, Master of Science

Utah State University, 2013

Major Professor: Dr. Tam Chantem

Department: Electrical and Computer Engineering

In intelligent transportation systems, most of the research work has focused on lane change assistant systems. No existing work considers minimizing the disruption of traffic flow by maximizing the number of lane changes while eliminating the collisions. In this thesis, we develop qualitative and quantitative approaches for minimizing the disruption of traffic flow for three lane scenarios and show that we can extend our approach to an arbitrary number of lanes. The proposed algorithm is able to achieve the maximum number of lane changes provided that only one vehicle per group (novel concept which is described in this thesis) is allowed to change lanes at a time. Simulation results show that our approach provides much better performance when compared with different lane change algorithms without incurring large overhead, and is hence suitable for online use. 


\section{Acknowledgments}

I would like to thank Dr. Tam Chantem for her support and assistance. I would also like to thank my friend, Sandeep Vundavalli, who helped me in the analysis of data.

Divya Desiraju 


\section{Contents}

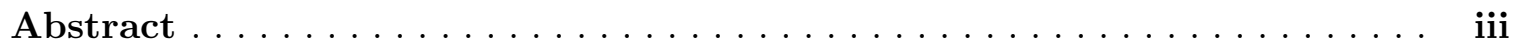

Public Abstract $\ldots \ldots \ldots \ldots \ldots \ldots \ldots \ldots \ldots \ldots \ldots \ldots \ldots \ldots \ldots$ iv

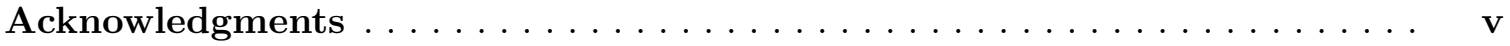

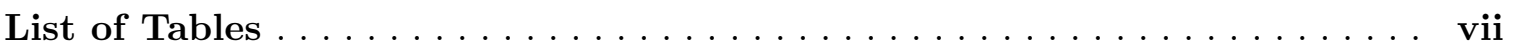

List of Figures $\ldots \ldots \ldots \ldots \ldots \ldots \ldots \ldots \ldots \ldots \ldots \ldots \ldots \ldots \ldots \ldots \ldots$

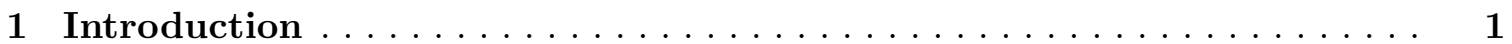

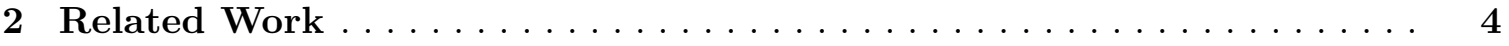

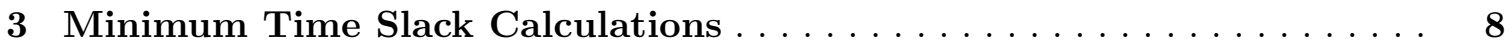

4 A Distributed Approach for Large Highways . . . . . . . . . . . . . . . 11

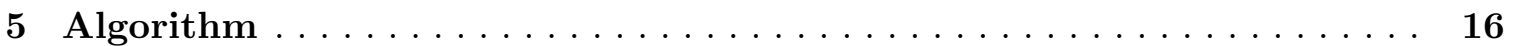

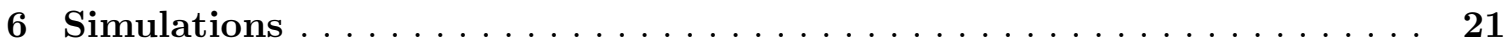

6.1 Simulation Framework . . . . . . . . . . . . . . . . . . . 21

6.2 Analytical Comparisons . . . . . . . . . . . . . . . . . . . 22

6.3 Simulation Results . . . . . . . . . . . . . . . . . . . . 24

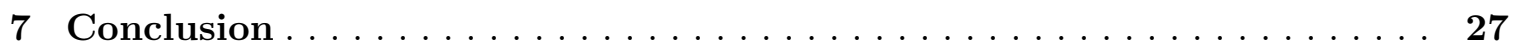

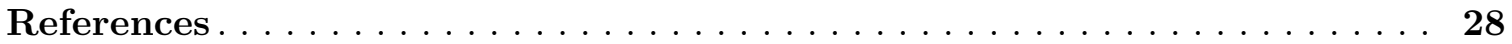




\section{List of Tables}

Table $\quad$ Page

6.1 The ranges used for the various attributes of the vehicles used in the simulations. 23

6.2 Worst case performance of different algorithms. . . . . . . . . . . . . 23

6.3 Best case performance of different algorithms. . . . . . . . . . . . . . . . 24

6.4 Minimum, maximum, and average percent improvement of our proposed approach over the baseline algorithms in terms of lane change ratio. . . . . . . 


\section{List of Figures}

Figure $\quad$ Page

2.1 An example 6 -lane highway. . . . . . . . . . . . . . . 6

2.2 Lane change maneuver of an automated vehicle. . . . . . . . . . . 7

3.1 An example scenario where $V_{i}$ wishes to change into $V_{j}$ 's lane. . . . . 8

3.2 If $V_{i}$ wishes to change into the top lane, it must consider its time slacks with respect to the shaded vehicles. . . . . . . . . . . . . . . .

4.1 An example used to illustrate how the lane change maximization problem on a 5-lane highway in each direction can be considered four lane-change maximization problems on three 3-lane highways and one 2-lane highway. The arrow in front of a vehicle indicates that vehicle's desire to perform a lane change. In Subproblem 1, only changes into the second lane are considered. This is the reason why the potential lane change by $V_{21}$ and $V_{23}$ are not considered in this subproblem. . . . . . . . . . . . . . .

5.1 Grouping examples. . . . . . . . . . . . . . . .

6.1 Simulation data. . . . . . . . . . . . . . . . 


\section{Chapter 1 Introduction}

Traffic congestion has become a major challenge for transportation professionals and roadway users across the world. As more of the world becomes more mobile, congestion during peak hours results in wasted time for billions of people around the globe. The effects

of congestion delays on the individual are mostly negative: there is a reduction of air quality due to vehicle idling and drivers' quality of life are affected by having large amount of nonproductive time which results in reduced time with family and friends, as well as economic losses due to non-productivity. Congestion also has a negative impact on safety, as it causes drivers to make increased decisions during stop and go traffic.

Financial, environmental, and land use considerations provide an increasingly difficult environment to significantly increase the capacity of roadways by adding additional lanes. Fortunately, congestion can be alleviated by replacing human-operated vehicles with automated vehicles, which free the driver from the mental workload of a large number of tasks, some of which have to be carried out in parallel [1]. The promise of reduced non-recurring congestion, due to reduction in vehicle crashes (approximately $25 \%$ of all congestion in the US), provides great opportunities for the supplement of automated vehicles into the fleet. In addition, computer-operated vehicles have shorter reaction times [2], which allow the vehicles to be closer to one another, thus increasing traffic flow.

Of all basic vehicular maneuvers, lane changing is arguably one of the most difficult ones. There were approximately 539,000 2-vehicle lane change crashes in the United States alone in 1999 [3]. Analysis of the German In-Depth Accident Study [3] from 1985 to 1999 shows that on average of more than $5 \%$ of accidents occurred while changing lanes. In 2008, $1.7 \%$ of the registered highway accidents in the Netherlands were caused by inadequate lane changing [4]. While it has been shown by Tsao et al. that the exit success percentage, which 
is the number of automated vehicles that successfully exit the system divided by the number of vehicles that need to exit, is well below $100 \%$ due to the lack of gaps sufficiently large for safe lane changes [5], we believe that it is crucial to provide a mechanism that best utilizes available gaps. To achieve the promise of high throughput and increased safety, a technique that minimizes the disruption of traffic flow by automated vehicles during lane changes must be implemented. In this article, we are interested in designing such a technique with the objective of maximizing the safe number of possible lane changes. Although there exists a large number of automated lane change assistant systems, as shown in Chapter 2, to the best of our knowledge, there has been no work that attempts to minimize the disruption of traffic flow by maximizing the number of lane changes for live traffic on a stretch of a highway with an arbitrary number of lanes, without any assumptions on vehicles' dynamic attributes such as speeds.

Our main contributions are as follows.

- Given an arbitrary number of automated vehicles, we design an algorithm to maximize the number of possible lane changes on an arbitrary segment of a highway at any given time. The proposed algorithm uses information such as vehicles positions, speeds, and time slacks (to be defined later) to make judicious lane change decisions without requiring prior knowledge on traffic patterns nor unnecessary braking. To reduce runtime overhead, we propose a distributed approach to allow for local lane changing decisions to be made during run time.

- We present a lane change simulation platform that enables the implementation and comparison of different lane change algorithms. A large number of simulations can be run efficiently and various simulation parameters such as the number of vehicles wishing to change lanes can be specified.

The remainder of the thesis is outlined as follows. We review existing literature regarding lane changes in Chapter 2. Chapter 2 also provides the system model and states the assumptions made in the thesis. The minimum time slack calculations, which is used to determine if a vehicle can change lanes without a collision, is presented in Chapter 3. 
Our distributed approach is discussed in Chapter 4 and the details of our online algorithm in Chapter 5. Chapter 5 also discusses the practical factors involved in implementing our approach in real operating scenarios. Simulation results are presented in Chapter 6 and Chapter 7 concludes the thesis. 


\section{Chapter 2 Related Work}

Lane changing is an important topic that has received significant research attention. Some work focuses on lane change assistant systems for human drivers $[4,6,7]$, while others consider lane change collision avoidance systems [8-12]. Chee and Tomizuka studied the lane change maneuver that is most comfortable to passengers $[13,14]$. The overtaking maneuver, which consists of one lane change from the right lane to the left lane and one lane change from the left to the right lane to pass a vehicle, has also been examined $[15,16]$.

Research has been conducted which yielded several sophisticated lane change controller designs [17-19]. A technique to perform lane changing to avoid obstacles is presented by Papadimitriou and Tomizuka [20]. A large number of automated lane change maneuver systems have been proposed to assist human drivers [21-26]. In particular, a neural network [24] and bayesian data fusion [25] approaches are used to enable safe lane changes. Jula et

al. presented some analysis to determine the minimum longitudinal spacing needed by a lane change [26].

In addition to increase passenger safety, several researchers have presented various models to predict a vehicle's intention of lane changing. For example, Xuan and Coifman exploited the availability of differential GPS data to detect lane change [27]. Angkititrakul et al. used a stochastic driver behavior to predict whether a lane change may occur [28]. Many cooperative approaches that make use of vehicles-to-vehicles (V2V) communications exist for a variety of lane change related purposes: eliminating risks during lane change [29], merging due to lane closures [30] and freeway entrance [31], overtaking assistance [32], and path predictions for increased safety [33]. Ardelt et al. proposed a probabilistic framework for automated vehicles on freeways [34].

Despite the wealth of research on lane change of automated vehicles, most work assume 
a 2-lane (in either direction) system, consider only one lane change at any given time, or assume that the vehicles travel at about the same speed [35-37]. Hilscher et al. presented a method to perform lane change safety verifications of an arbitrary number of automated vehicles on multi-lane highway [38], but do not attempt to maximize the number of lane changes at a given time.

A closely related topic to lane changing is the lane assignment problem where automated vehicles are assigned to their given lanes for each segment of the highway. Hall et al. formulated this scheduling problem as a linear optimization problem with the objective of throughput maximization $[39,40]$, while Ramaswamy et al. opted to minimize travel time [41]. Lane assignments may be made for each vehicle or a group of vehicle and usually depend on the distance a vehicle has to travel before exiting the highway. The lane assignment problem can also be solved using genetic algorithms, as shown by Kim et al. [42]. The key assumptions made in previous work is that (i) the automated vehicles travel at about the same speed for a given lane or across all the lanes, and/or that this speed does not depend on traffic volume, and (ii) traffic is known a priori. In contrast, we do not rely on neither of these assumptions in this work.

We consider a set of automated vehicles $\Psi$ along an arbitrary segment of an $m$-lane highway, where $m$ is an integer and $m \geq 2$. The width $W$ of each lane is known a priori. Although we assume, for the sake of simplicity, that all lane widths are equal, this work can readily be applied to highways in which lane widths differ. Each automated vehicle $V_{i}$ is characterized by its length $l_{i}$ and width $w_{i}$. At any given time, the current lane, velocity $u_{i}$, acceleration $a_{i}$ are known. In addition, the position $p_{i}$ of the front left of the vehicle with respect to some reference point, which is represented by a tuple $\left(x_{i}, y_{i}\right)$, is known for vehicle $V_{i}$. Figure 2.1 shows a 6-lane highway example with three automated vehicles. At any point in time, a vehicle may wish to perform a lane change for whatever reason. For instance, a vehicle $V_{i}$ may want to change lane since it is coming upon a slower moving vehicle $V_{j}$ in front of it.

In such a case, if a lane change is not made (or not made until later), $V_{i}$ will slow down 

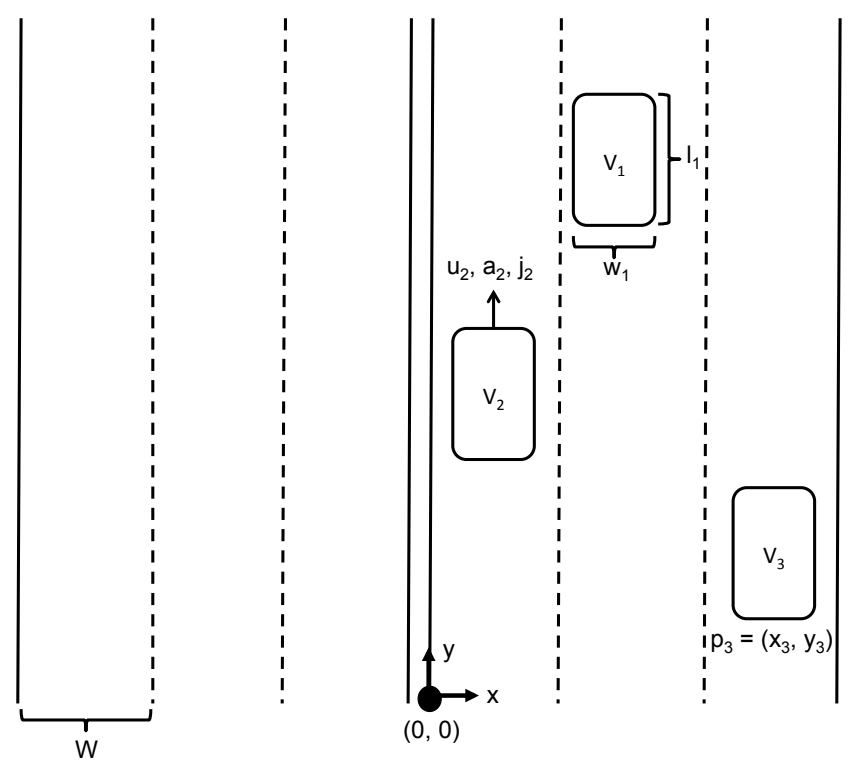

Fig. 2.1: An example 6-lane highway.

and adopt the Gipps' car following model [43], which is a widely used car following model. That said, our approach can be modified for use with other car following models.

We assume the existence of either a roadside infrastructure, which allows for vehicleto-infrastructure (V2I) communications [44,45], or a vehicular adhoc network (VANET) for vehicle-to-vehicle (V2V) communications [33]. Such communications are used by a vehicle to obtain necessary information (e.g., velocity, acceleration, etc.) of other vehicles in the vicinity.

The distance traveled by a vehicle $V_{i}$ during the time interval $\left[t_{0}, t\right]$ is

$$
s_{i}(t)=s_{i}\left(t_{0}\right)+u_{i}\left(t-t_{0}\right)+\frac{1}{2} a_{i}\left(t-t_{0}\right)^{2}+\frac{1}{6} j_{i}\left(t-t_{0}\right)^{3} .
$$

In this article, we adopt the approach used by Neades and Ward [46] to compute the time a vehicle $V_{i}$ requires to perform a lane change. Specifically, the objective of the original analysis is to compute the minimum time taken to change lanes given the critical speed, which is the maximum speed at which a turn can be negotiated [46]. Significant modifications were made to the original derivation to obtain the time required to perform a lane change for a given vehicle with arbitrary velocity and acceleration. That is, the swerve 
taken by vehicle $V_{i}$ follows the trajectory (dotted line) illustrated in Figure 2.2. Here, $a$ is assumed to be half the width of a lane and thus is known. The angle $\theta_{i}$ is also known since we are considering automated vehicles.

With known values of $\theta_{i}$ and $a_{i}, c_{i}=\frac{a_{i}}{\cos \left(\theta_{i}\right)}$. Applying Pythagorean theorem, $b_{i}=$ $\sqrt{\left(c_{i}^{2}-a_{i}^{2}\right)}$. The total distance vehicle $V_{i}$ requires to perform a lane change (i.e., complete swerve) is $d_{i}=2\left(\frac{\pi}{2} b_{i}\right)=\pi b_{i}$. Finally, the time to lane change $t_{i}^{c}$ for vehicle $V_{i}$ can be found by solving the following equation

$$
\pi b_{i}=u_{i} t_{i}^{c}+\frac{1}{2} a_{i}\left(t_{i}^{c}\right)^{2}+\frac{1}{6} j_{i}\left(t_{i}^{c}\right)^{3}
$$

For the sake of clarity, we ignore lateral acceleration. However, said acceleration can be incorporated when calculating the time to lane change. The proposed technique requires no modification when lateral acceleration is considered.

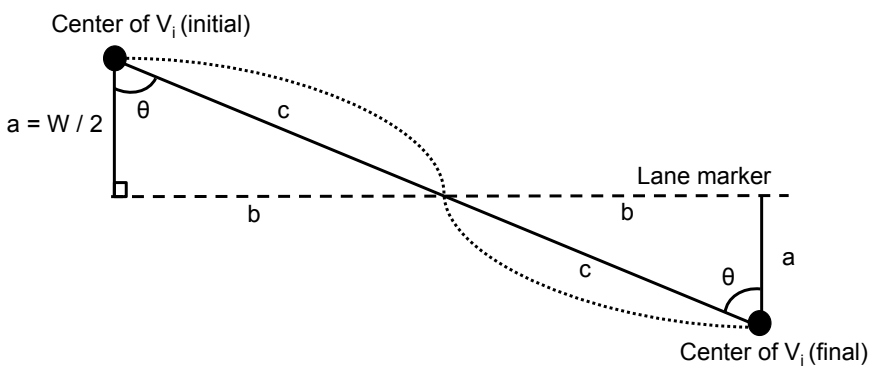

Fig. 2.2: Lane change maneuver of an automated vehicle. 


\section{Chapter 3}

\section{Minimum Time Slack Calculations}

Let us consider an automated vehicle $V_{i}$ whose attributes are as described in Chapter 2. As shown in Chapter 2, the time to lane change for $V_{i}$ can be computed as in Equation (2.2) and depends on a number of factors such as $V_{i}$ 's speed, as well as the lane width. However, since $V_{i}$ is unlikely to be the only vehicle on a given stretch of highway, $V_{i}$ may not be able to change lanes right away or a collision may ensue if the gap between $V_{i}$ and another vehicle is not large enough. We now use a simple example to demonstrate how the time vehicle $V_{i}$ has to change lane can be calculated.

Figure 3.1 shows an example scenario consisting of two automated vehicles $V_{i}$ and $V_{j}$ at some time $t$. Let the current positions of $V_{i}$ and $V_{j}$ be $p_{i}=\left(x_{i}, y_{i}\right)$ and $p_{j}=\left(x_{j}, y_{j}\right)$, respectively. In addition, $V_{i}$ is in front of $V_{j}$, (i.e., $y_{i} \geq y_{j}$ ). Let us assume $V_{i}$ starts the lane change process at time $t$ and both vehicles maintain their velocities and accelerations. Let $p_{i}^{\prime}=\left(x_{i}^{\prime}, y_{i}^{\prime}\right)$ be the new position of $V_{i}$ at time $t+t_{i}^{c}$. In addition, let $V_{j}^{\prime}$ 's position at time $t+t_{i}^{c}$ be $p_{j}^{\prime}=\left(x_{j}^{\prime}, y_{j}^{\prime}\right)$. Clearly, $x_{j}^{\prime}=x_{j}=x_{i}^{\prime}$, and

$$
y_{j}^{\prime}=y_{j}+u_{j}\left(t_{i}^{c}\right)+\frac{1}{2} a_{j}\left(t_{i}^{c}\right)^{2}+\frac{1}{6} j_{j}\left(t_{i}^{c}\right)^{3} .
$$

A collision will not occur if, at time $t+t_{i}^{c}, V_{j}$ either remains behind $V_{i}$ and the latter's headway is at least three seconds or $V_{j}$ is now in front of $V_{i}$ and its headway is at least

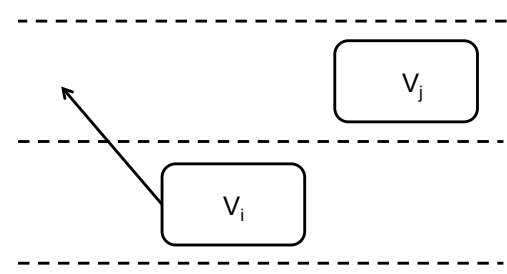

Fig. 3.1: An example scenario where $V_{i}$ wishes to change into $V_{j}$ 's lane. 
three seconds. For the first scenario to be true, the following must be satisfied

$$
y_{i}^{\prime}-l_{i} \geq r\left(v_{i}, a_{i}, j_{i}\right)+y_{j}^{\prime},
$$

where $l_{i}$ is the length of $V_{i}$ and $r\left(v_{i}, a_{i}, j_{i}\right)$ is the minimum distance between $V_{i}$ and $V_{j}$ according to the 3 -second following distance rule, which depends on $v_{i}, a_{i}$, and $j_{i}$. Similarly, if $V_{j}$ is now in front of $V_{i}$, we have

$$
y_{j}^{\prime}-l_{j} \geq r\left(v_{j}, a_{j}, j_{j}\right)+y_{i}^{\prime} .
$$

Consequently, $t_{i, j}^{h}$, the time $V_{i}$ has to change lane with respect to $V_{j}$, can be obtained by solving the following expression

$$
y_{i}^{\prime}-l_{i}-r\left(v_{i}, a_{i}, j_{i}\right)=u_{j}\left(t_{i, j}^{h}\right)+\frac{1}{2} a_{j}\left(t_{i, j}^{h}\right)^{2}+\frac{1}{6} j_{j}\left(t_{i, j}^{h}\right)^{3},
$$

provided that $V_{i}$ will end up in front of $V_{j}$. A similar condition can be derived for the case where $V_{j}$ will be in front of $V_{i}$.

We are now ready to define the time slack of $V_{i}$ with respect to $V_{j}$.

Definition 1 The time slack of $V_{i}$ with respect to $V_{j}$ is the difference between the time $V_{i}$ has to change lane with respect to $V_{j}$ and the time $V_{i}$ takes to change lane given its current velocity and acceleration. In other words,

$$
s l_{i, j}=t_{i, j}^{h}-t_{c}^{i}
$$

The time slack helps to determine whether a lane change is safe. That is, a positive time slack denotes a safe lane change (with respect to another vehicle) while a negative time slack implies that a collision may occur. In real scenarios, a vehicle wanting to change lane may need to consider its time slacks with respect to a number of vehicles, instead of just one vehicle. Figure 3.2 indicates the vehicles that $V_{i}$ (the vehicle wanting to change lanes) 


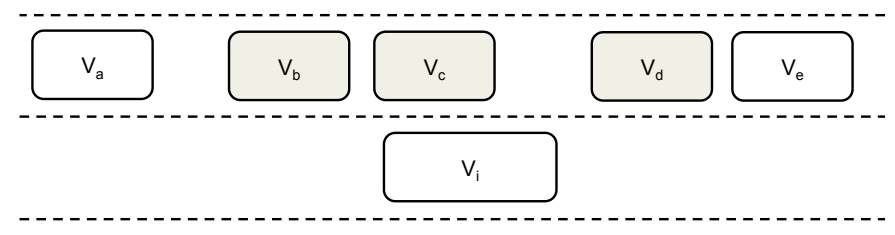

Fig. 3.2: If $V_{i}$ wishes to change into the top lane, it must consider its time slacks with respect to the shaded vehicles.

needs to account for. Let $\Gamma$ be the set of vehicles currently in the lane that $V_{i}$ wishes to change to. Then, the time slack of $V_{i}$ with respect to $V_{j} \in \Gamma$ needs to be computed if

- $V_{j}$ laterally overlaps with $V_{i}$, (i.e., $y_{i}-l_{i} \leq y_{j} \leq y_{i}$ or $y_{i}-l_{i} \leq y_{j}-l_{j} \leq y_{i}$ );

- $V_{j}$ is the lateral vehicle immediately in front of $V_{i}$, (i.e., $y_{j}=\min _{V_{k} \in \Gamma}\left\{y_{k}\right\} \mid y_{j}>y_{i}$ ) and $V_{j}$ is not traveling faster than $V_{i}$; or

- $V_{j}$ is the lateral vehicle immediately behind $V_{i}$, (i.e., $y_{j}=\max _{V_{k} \in \Gamma}\left\{y_{k}\right\} \mid y_{j}<y_{i}-l_{i}$ ).

We are now ready to generalize the concept of time slack.

Definition 2 The minimum time slack of $V_{i}$ with respect to a group of vehicles $\Gamma^{\prime}$ is the minimum difference between the time $V_{i}$ has to change lane with respect to $V_{j} \in \Gamma^{\prime}$ and the time $V_{i}$ takes to change lane given its current velocity and acceleration. In other words,

$$
s l_{i}^{*}=\min _{V_{j} \in \Gamma^{\prime}} s l_{i, j} .
$$

If at most one vehicle wants to change lanes, a positive minimum time slack indicates that a safe lane change can take place. We next consider the more realistic scenarios where more than one vehicle on a segment of a highway may wish to change lanes. 


\section{Chapter 4}

\section{A Distributed Approach for Large Highways}

One way to maximize the number of lane changes given a set of automated vehicles on a stretch of highway is to formulate the problem as an optimization problem with constraints on safety for each time instant. However, the resultant optimization problem is relatively complex and contains integer variables, making it hard to solve the problem efficiently online using a mixed-integer programming solver. An alternative approach is to consider, for each stretch of the highway of interest, all the vehicles in all the lanes in order to make centralized, globally optimal decisions. However, this approach may not be practical or efficient enough when there is a large number of vehicles. In addition, such a centralized approach requires that each vehicle be aware of all other vehicles on that particular stretch of a highway, even if they are far enough apart that they cannot possibly interfere with one another. For these reasons, we resort to designing efficient local algorithms. The key idea is to solve the problem in a distributed manner instead of globally.

We observe that given an m-lane highway in each direction, we can divide the problem of lane change maximization into a number subproblems, as illustrated in Figure 4.1. In this example, there are 5 lanes and 16 vehicles, 8 of which wish to change lane. To reduce runtime overhead, a subproblem is created for each lane that at least one vehicle wants to change to. There are four subproblems in this example, as no vehicle wishes to change to the top lane. In subproblem 1 (Figure 4.1(b)), potential changes into the second lane from the top are considered. For this reason, potential lane changes by $V_{21}$ and $V_{23}$ are ignored since these vehicles may or may not change lane in the end. This process is repeated for all the lanes. Algorithm 4.1 provides the steps needed to create the subproblems. It takes as inputs the number of lanes and the set of vehicles, and returns a set of subproblems. Each subproblem consists of a number of lanes, the vehicles in each of the lanes, and a set of vehicles that wish to change into a common lane. 


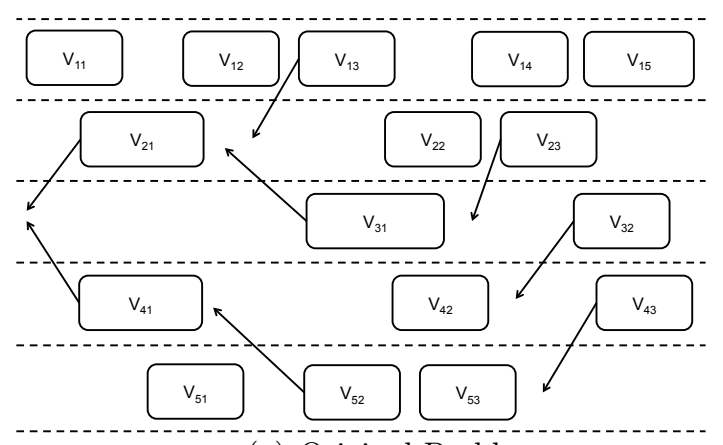

(a) Original Problem

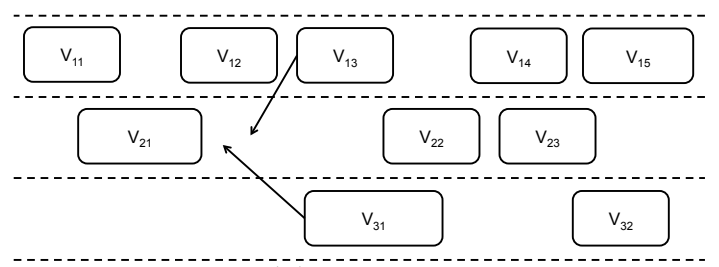

(b) Subproblem 1

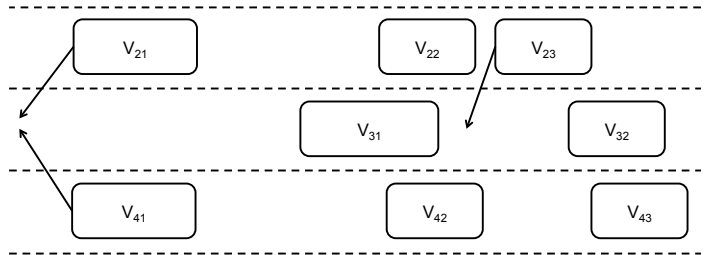

(c) Subproblem 2

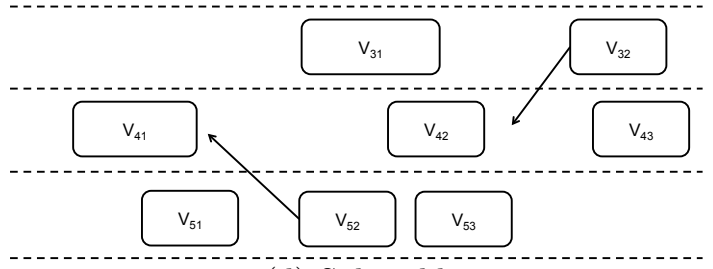

(d) Subproblem 3

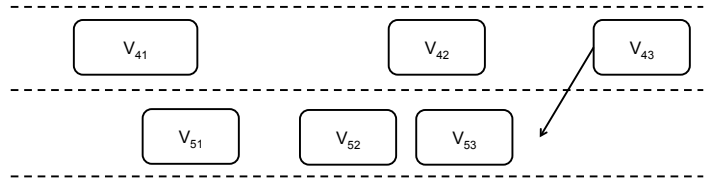

(e) Subproblem 4

Fig. 4.1: An example used to illustrate how the lane change maximization problem on a 5-lane highway in each direction can be considered four lane-change maximization problems on three 3-lane highways and one 2-lane highway. The arrow in front of a vehicle indicates that vehicle's desire to perform a lane change. In Subproblem 1, only changes into the second lane are considered. This is the reason why the potential lane change by $V_{21}$ and $V_{23}$ are not considered in this subproblem. 


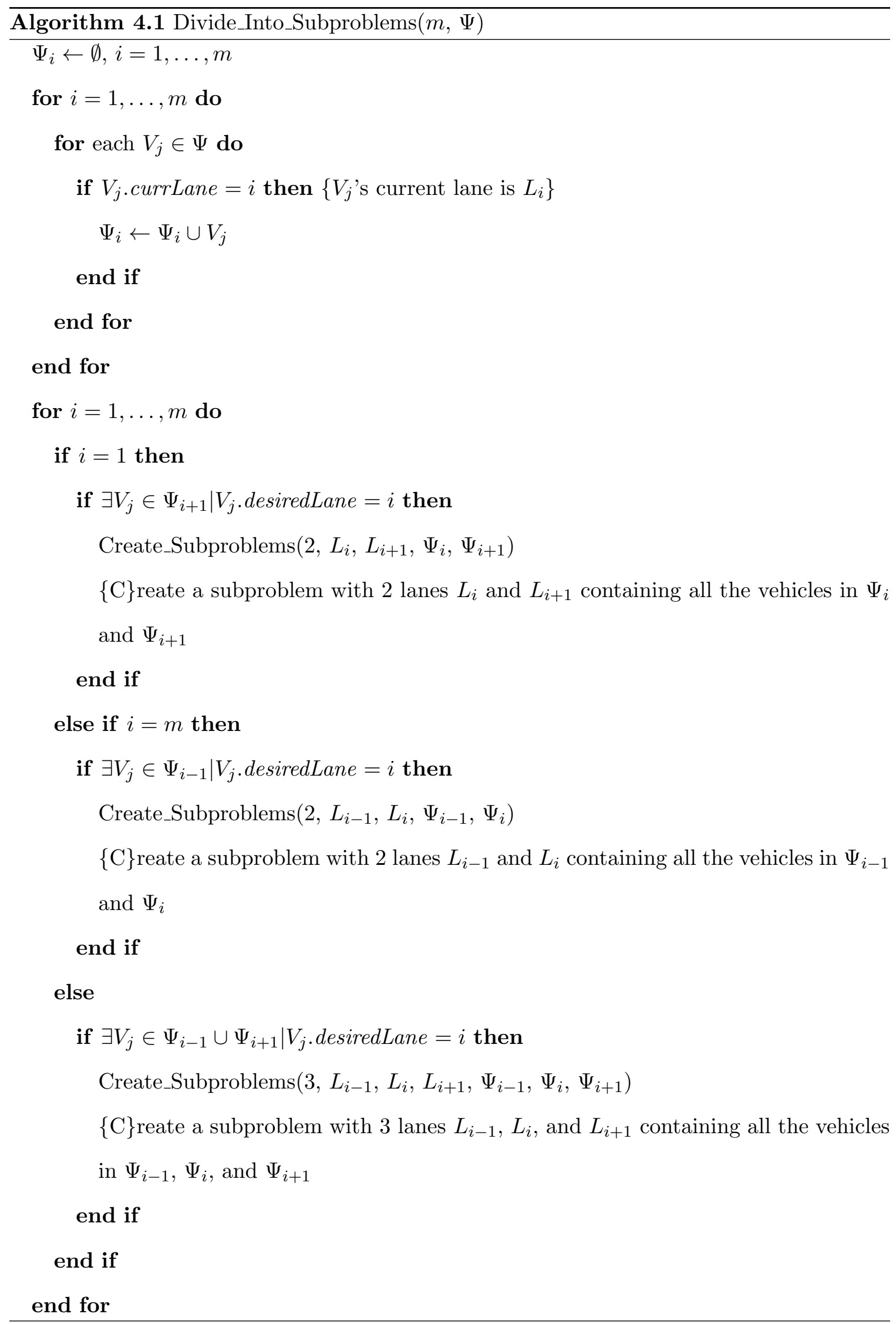


The time complexity of Algorithm 4.1 is $O(m \cdot|\Psi|)$, where $m$ is the number of lanes on the stretch of the highway under consideration and $|\Psi|$ is the number of vehicles associated with said stretch of the highway. To prove some properties of the subproblems created using Algorithm 4.1, we start with a definition followed by a lemma.

Definition 3 A feasible lane change configuration within a subproblem is a set of lane change decisions made within that subproblem that ensures no collision among vehicles within the subproblem will occur.

Lemma 1 Consider an m-lane highway in each direction, a set of automated vehicles $\Psi$, and a set of automated vehicles wanting to change lane $\Lambda$ where $\Lambda \subseteq \Psi$. Applying Algorithm 4.1 will result in at most $m$ subproblems. In addition, decisions whether or not to allow vehicles in each subproblem to change lane can be made independently, (i.e., without considering decisions made in other subproblems) and no collision will occur due to these independent lane change decisions as long as the lane change configuration within each subproblem is feasible.

Proof: It is straightforward to show that there can be at most $m$ subproblems, since there can be at most one subproblem per lane. We now show that no collision can occur by making lane change decisions for each subproblem in parallel.

Without loss of generality, let us assume that there are two subproblems $S_{1}$ and $S_{2}$ for changes into lanes $L_{1}$ and $L_{2}$, respectively. In addition, a feasible lane change configuration within each subproblem is found, (i.e., there no collisions among vehicles within the subproblem). Now, let us assume that applying said feasible lane change configurations result in a collision. Since, by definition, a feasible lane change configuration ensures no collision among vehicles within a subproblem can happen, a collision must occur outside of the subproblems, (i.e., in the original problem). Since subproblem $S_{1}$ focuses on changes into lane $L_{1}$ and subproblem $S_{2}$ lane $L_{2}$, a collision can only occur if a vehicle from lane $L_{1}$ does not safely change into lane $L_{2}$ (or vice versa). However, during the creation of the subproblems, all the vehicles in a given lane are considered. Hence, a collision cannot happen. This is a contradiction and the lemma is proved. 
Based on the above lemma, we will now focus on the problem of maximizing the number of lane changes on a 3-lane highway. 


\section{Chapter 5}

\section{Algorithm}

We are interested in solving the following problem.

Problem 1: Given a 3-lane highway with a set of automated vehicles whose attributes such as velocity and acceleration are known, and in which a subset of those vehicles wish to change lane, determine the set of vehicles that are allowed to change lanes in order to maximize the total number of lane change at any given time.

Although it has been shown in the previous section that an m-lane highway can be divided into several 3-lane highways to reduce the complexity of the problem, the number of automated vehicles on a given stretch of a highway may still be large. To further optimize for the efficiency of our approach, we now introduce the concept of grouping of vehicles, which will allow us to solve Problem 1 in a distributed manner.

The main idea behind grouping is based on the observation that several lane changes may occur at the same time on a given stretch of a 3-lane highway, as long as vehicles are far enough apart, as shown in Figure 5.1(a). This idea can be taken a step further, as illustrated in Figure 5.1(b), by observing that grouping can be made with respect to some vehicle. For example, in Figure 5.1(b), $V_{A}$ can change lane without needing to consider $V_{D}$, but must account for both $V_{B}$ and $V_{C}$, as the latter vehicles are within its "range." Our concept of grouping allows for only for a small group of vehicles to be considered for simultaneous lane change decisions. We now present our grouping algorithm, which is shown in Algorithm 5.1.

Algorithm 5.1 takes as input $\Psi$, which the set of vehicles on a 3-lane highway. The first step taken by Algorithm 5.1 is to sort the vehicles such that $\forall V_{i}, V_{j} \in \Psi, i<j$ if and only if $y_{i}<y_{j}$. In other words, vehicles are sorted in a non-increasing order of their $y$ positions. Algorithm 5.1 then starts a group containing $V_{i}$, which is the first vehicle in $\Psi$. Next, using $V_{i}$ 's time to change lane $t_{c}^{i}$, it computes the distance separating $V_{i}$ and $V_{j}$ (the 


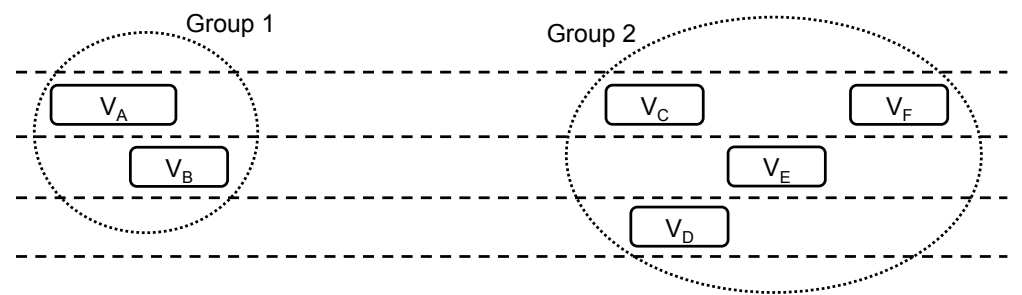

(a) If $V_{A}$ and $V_{B}$ are far enough apart from the rest of the vehicles, they can be considered separately from the other vehicles when making lane change decisions.

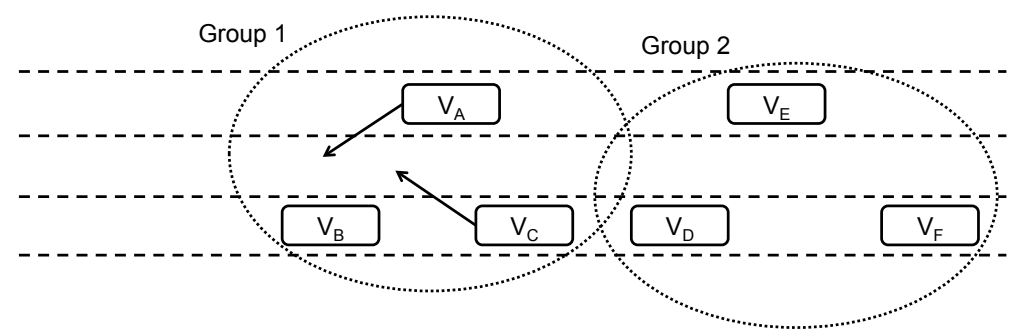

(b) Here, $V_{A}$ must consider $V_{B}$ and $V_{C}$ but can ignore $V_{D}$.

Fig. 5.1: Grouping examples.

next vehicle in $\Psi$ ), including the 3 -second following distance rule. If this distance $d_{s}$ is negative, a collision may occur if $V_{i}$ and $V_{j}$ change lanes at the same time. As a result, $V_{j}$ must be grouped with $V_{i}$ and Algorithm 5.1 continues the same process with the next vehicle in $\Psi$. Otherwise, the current grouping is finished and the new group is started until there are no vehicles remaining in $\Psi$. An optimization can be made to Algorithm 5.1 by only including vehicles that wish to change to the common lane and the vehicles already in that lane. This is because vehicles that do not currently wish to change lanes and which are not currently in the common lane cannot interfere with those wishing to switch lanes.

The time complexity of Algorithm 5.1 is $O\left(|\Psi|^{2}\right)$, since sorting takes $O(|\Psi| \cdot \log |\Psi|)$ and the most time consuming part of the algorithm occurs within the while loop. In the worst case, one vehicle is removed from $\Psi$ in every iteration, which means that the while loop will iterate for at most $|\Psi|$ times. In addition, the inside while loop will iterate for at most $|\Psi|$ times, while all other operations take constant time. The time complexity of Algorithm 5.1 can be reduced to $O(|\Psi| \cdot \log |\Psi|)$ by replacing the inner while loop with a for loop and using binary search. 


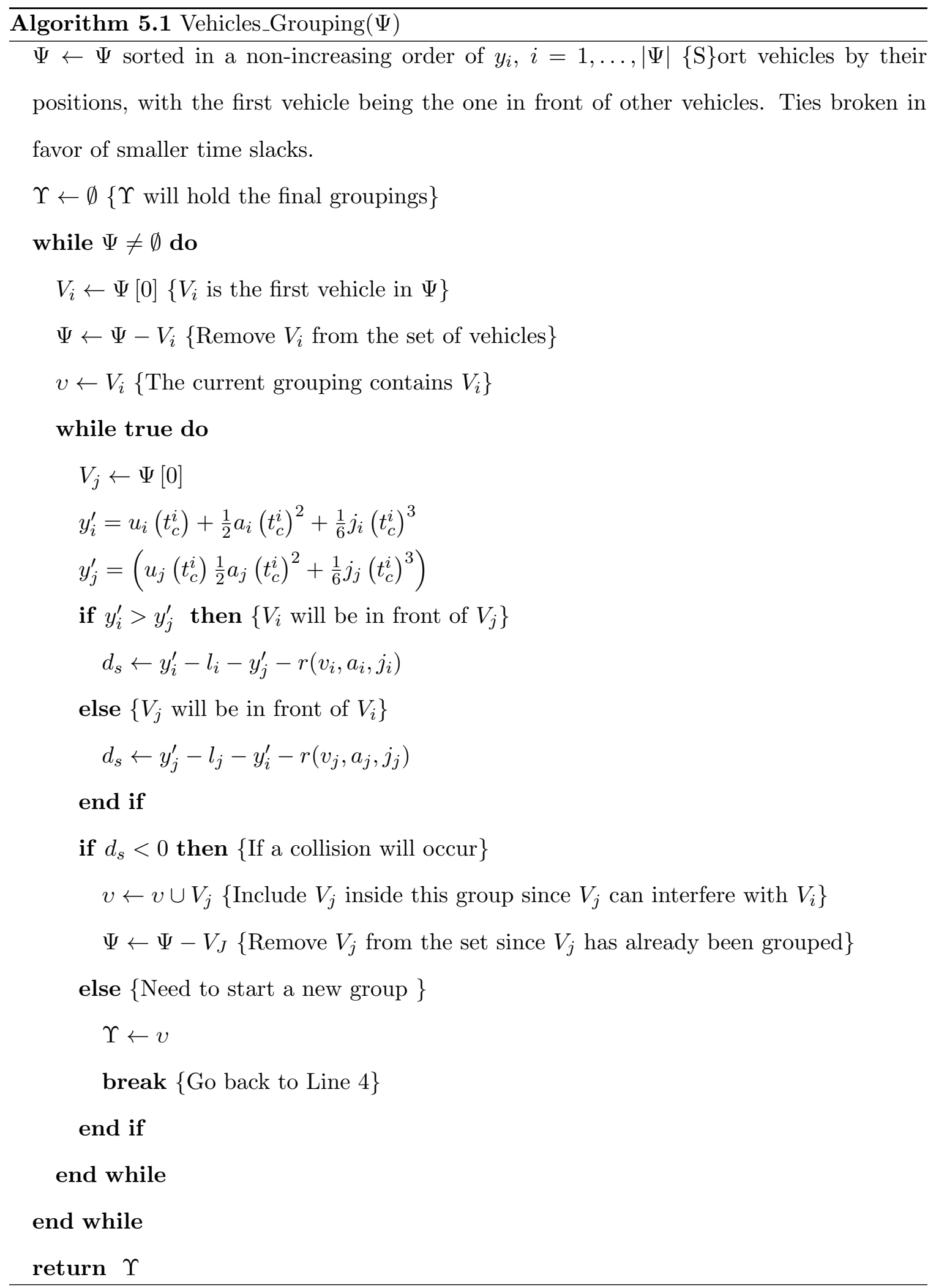


It is worth noting that some checkpoints are left off the description of Algorithm 5.1 for the sake of clarity. For example, additional steps are needed if there exist at least two vehicles with exactly the same y values, i.e., $\exists y_{i}=y_{j}, V_{i}, V_{j} \in \Psi$.

Once grouping takes place, the vehicle at the front of each group will be selected for lane change. We now discuss some properties of Algorithm 5.1 using the following lemmas and theorem.

Lemma 2 Consider a 3-lane highway with a set of automated vehicles $\Psi$. If Algorithm 5.1 is used to group vehicles in such a way that one vehicle per group performs a lane change, no collisions will take place.

Proof: The proof is straightforward, as a new group is formed by Algorithm 5.1 if the safety distance computed on Line 9 is satisfied.

Lemma 3 Consider a 3-lane highway with a set of automated vehicles $\Psi$, applying Algorithm 5.1 results in the maximum number of groups where one vehicle per group can change lane without violating safety constraints.

Proof: We prove the lemma using contradiction. Let us suppose that Algorithm 5.1 found $n$ groups, but that a feasible solution with $n+1$ groups exists. Without loss of generality, let us also assume that in the second, (i.e., better set of solutions), the vehicles in the $n^{\text {th }}$ and $n+1^{\text {th }}$ groups make up the $n^{\text {th }}$ group found by Algorithm 5.1. This means that it is possible to divide the $n^{\text {th }}$ group found by Algorithm 5.1 into two (or more) groups. However, in Algorithm 5.1, a new group is form only if the safety constraint (Line 9) is satisfied. This violates the original assumption that the second set of solution is feasible. Hence, the lemma is proved.

Theorem 1 Consider a 3-lane highway with a set of automated vehicles $\Psi$, some of which wish to switch to the center lane. Using Algorithm 5.1 to group the vehicles and selecting the vehicle at the front of each group for lane change results in the maximum number of lane changes, provided that only one vehicle per group is allowed to change lane at a given time instant. 
Proof: The proof directly follows from Lemmas 2 and 3.

Algorithms 4.1 and 5.1 were described in such a way as to facilitate the discussions. The use of Algorithm 4.1 in real operating scenarios is straightforward; the "center" lane is always the lane vehicles wish to change to. Hence, for an m-lane highway (in each direction), there can logically be up to six "center" lanes.

As for Algorithm 5.1, information regarding groups must be passed downstream, (i.e., from vehicles in the front to the ones in the back). However, the process can be optimized whenever situations similar to the one in Figure 5.1(a) arise. That is, since $V_{C}$ can obtain information regarding the position, velocity and acceleration of $V_{B}, V_{C}$ can easily determine if it can form its own group that is separate from $V_{B}$. 


\section{Chapter 6 Simulations}

We compare the effectiveness and efficiency of our proposed algorithm against the following techniques, both analytically and using simulations. Note that comparison choices are very limited, as we are the first to consider the problem of lane change maximization. To ensure fair comparison, an m-lane highway (in each direction) is divided into several 3-lane highways as discussed in Chapter 4.

- Random algorithm: A number between $[0, k]$ is randomly generated, where $k$ is the number of vehicles that wish to make a lane change. Based on this random number $r, r$ vehicles will randomly be selected for lane change.

- Greedy algorithm: In this algorithm, the minimum time slacks are ignored and all the vehicles that want to change lane will be allowed to change lane.

- Least slack first algorithm: One vehicle is selected to change lane at any point in time. The vehicle with the minimum time slack will be chosen.

\subsection{Simulation Framework}

Since the objective of the simulations is to evaluate the performance of the proposed algorithm compared to the baseline algorithms, we assume that information on surrounding vehicles such as positions, velocities, and accelerations are readily available. (The information would in reality be sent to the vehicles using either V2V or V2I.) Specifically, for each vehicle in a given time instant, the following values are known to the system: unique vehicle ID, position, velocity, acceleration, safe distance, with respect to the vehicle immediately in front of it, according to the 3 -second rule, $\theta$ (the angle at which the vehicle takes to perform a lane change, see Chapter 2), time taken to perform a lane change, current lane, 
and desired lane. If a vehicle does not wish to change lanes at this time, then the current lane is the same as the desired lane.

We randomly generated 20,000 benchmarks, each of which contains a number of automated vehicles on a 3-lane highway in each direction. The highway is assumed to have three lanes since we have previously shown that the problem of lane change maximization on wider highways can be divided into a number of subproblems with 3-lane highways. The total number of vehicles in a benchmark ranges from 5 to 100, with the number of vehicles wishing to change lane being between 0 and 55. For the sake of simplicity, all vehicles are assumed to have the same width, length, and $\theta$ set to zero. The positions, velocities, accelerations, as well as starting and end lanes, were randomly generated. The ranges for these values can be found in Table 6.1. Given these values, the safety distance (the minimum distance separating this vehicle from the vehicle directly in front of it) and the time the vehicle takes to change lane, can be computed.

The following performance metrics will be used in each benchmark to assess the performance of our algorithm: lane change ratios, collision ratios, and time overheads. The lane change ratio $l$ is defined as

$$
l=\frac{\text { Number of safe lane changes performed }}{\text { Total number of desired lane changes }},
$$

while the collision ratio $c$ can be expressed as

$$
c=\frac{\text { Number of collisions }}{\text { Total number of vehicles }} \text {. }
$$

Finally, the time overheads represent the overheads associated with all the algorithms and will indicate whether our proposed method is suitable for online use.

\subsection{Analytical Comparisons}

Before presenting the simulation results, we analytically derive the best- and worst-case scenarios for the algorithms. As will be shown in the next section, the simulation results 
Table 6.1: The ranges used for the various attributes of the vehicles used in the simulations.

\begin{tabular}{|c|c|c|}
\hline Vehicle Attribute & Minimum Value & Maximum Value \\
\hline Y-Position & 0 & 1600 \\
Velocity $(\mathrm{m} / \mathrm{s})$ & 5 & 30 \\
Acceleration $\left(\mathrm{m} / \mathrm{s}^{2}\right)$ & 0 & 2 \\
\hline
\end{tabular}

verify the analysis presented here.

Let $k$ and $n$ be the number of vehicles that wish to change lane and the number of groups when using the proposed algorithm, respectively. The best and worst cases are shown in Tables 6.2 and 6.3, respectively. Thanks to our grouping method, no collisions will occur. The proposed algorithm results in the maximum number of lane changes, provided that at most one vehicle per group can change lane. For both the random and greedy algorithms, the worst case occurs when every lane change results in a collision $(r$ is the random number generated by the random algorithm and represents the number of vehicles allowed to change lanes using that algorithm). In contrast, the least-slack first algorithm ensures that exactly one safe lane change is performed at any point in time.

The best-case scenarios for the proposed algorithm and the least-slack first algorithm are the same as in the worst-case scenarios. In the best case, using the random and greedy algorithms will result in no collisions. Clearly, our proposed technique never performs worse than the other algorithms and has a much better performance in the worst-case scenario.

Table 6.2: Worst case performance of different algorithms.

\begin{tabular}{|c|c|c|}
\hline Algorithm & Number of collisions & Number of safe lane changes \\
\hline Proposed & 0 & $n, 1 \leq n \leq k$ \\
Random & $r$ & 0 \\
Greedy & $k$ & 0 \\
Least-Slack First & 0 & 1 \\
\hline
\end{tabular}


Table 6.3: Best case performance of different algorithms.

\begin{tabular}{|c|c|c|}
\hline Algorithm & Number of collisions & Number of safe lane changes \\
\hline Proposed & 0 & $k$ \\
Random & 0 & $r$ \\
Greedy & 0 & $k$ \\
Least-Slack First & 0 & 1 \\
\hline
\end{tabular}

\subsection{Simulation Results}

The average lane change ratio for the different algorithms is shown in Figure 6.1(a). It is clear from the plots that our proposed algorithm outperforms the baseline algorithms by significant margins. The maximum, minimum, and average percent improvements in lane change ratio of our method over the other algorithms are shown in Table 6.3. Figure 6.1(b) depicts the average collision ratio for the algorithms. Both our method and the least-slack first algorithm resulted in no collisions, while, as expected, the greedy algorithm has the highest collision ratio.

From the below data, it is clear that our proposed method achieves the best performance in terms of lane changes and collision avoidance. The average time overhead of our algorithm compared to the other methods is shown in Figure 6.1(c) based on the simulations conducted on an Intel i7 $3.50 \mathrm{GHz}$ with $16 \mathrm{~GB}$ memory. Since our algorithm is the most sophisticated, it is also the most time consuming approach.

To recap, the simulation data shows that our proposed method can efficiently and effective manage gaps between vehicles to allow for as many vehicles that need to change lanes to do so without causing collisions. We intend to improve the efficiency of our algorithm in future work. That said, the method presented in this thesis is appropriate for small to mid-size lane change scenarios. 


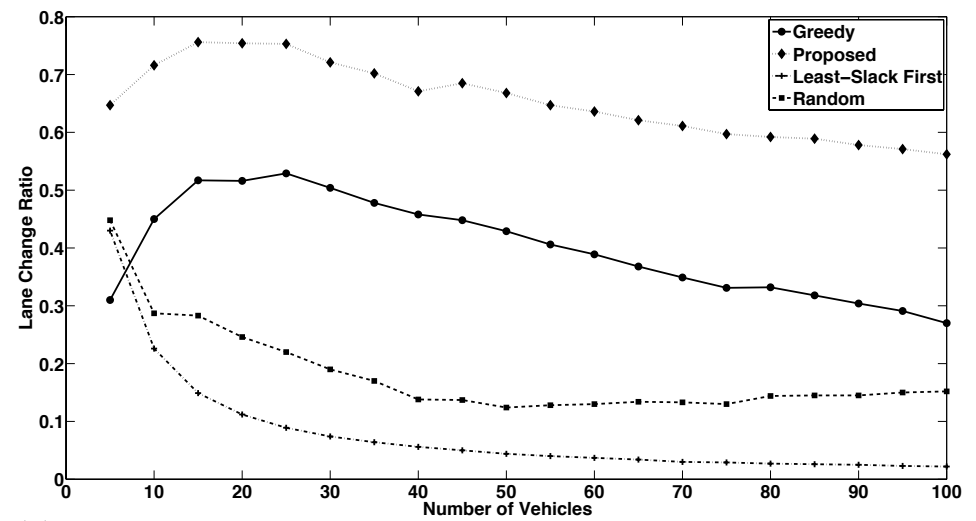

(a) Average lane change ratio as a function of number of vehicles for the different algorithms.

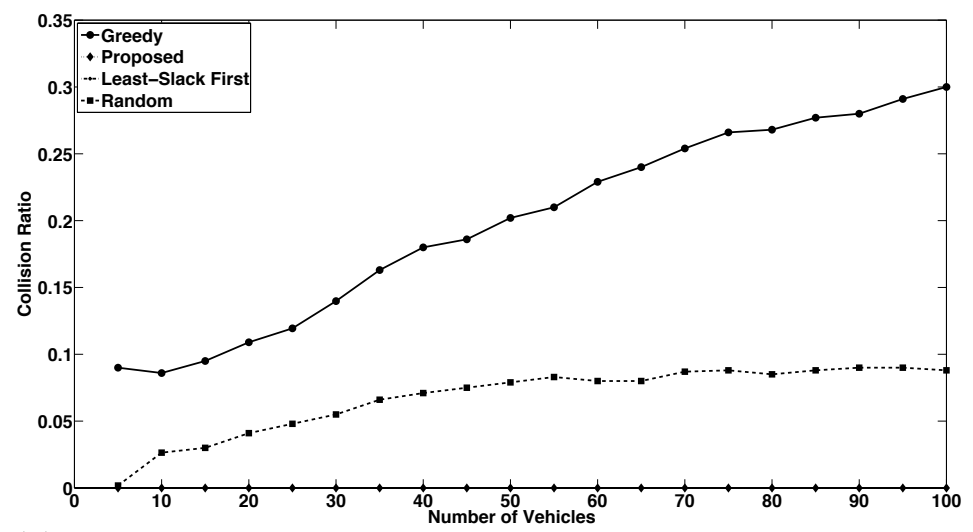

(b) Average collision ratio as a function of number of vehicles for the different algorithms.

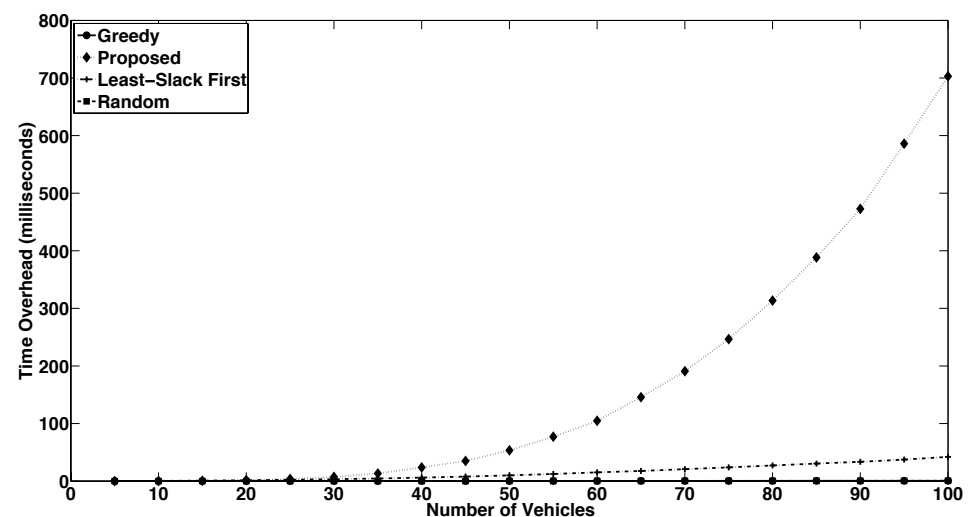

(c) Average time overhead in seconds as a function of number of vehicles for the different algorithms.

Fig. 6.1: Simulation data. 
Table 6.4: Minimum, maximum, and average percent improvement of our proposed approach over the baseline algorithms in terms of lane change ratio.

\begin{tabular}{|c|c|c|c|}
\hline \% Improv. on Lane Change Ratio & Minimum & Maximum & Average \\
\hline Greedy & 42.3 & 108.7 & 67.6 \\
Least-Slack First & 50.5 & 2454.5 & 1385.8 \\
Random & 44.4 & 438.7 & 298.5 \\
\hline
\end{tabular}




\section{Chapter 7 Conclusion}

This thesis discussed the problem of lane change maximization of automated vehicles in order to minimize the disruption of traffic flow caused by lane changes. A distributed algorithm was proposed to solve the problem. The key ideas behind said algorithm are time slack calculations and the concept of vehicle grouping. Simulation results show that the proposed method increases the number of lane changes by up to 109-2454\% and 68-1386\% on average compared to a number of baseline algorithms.

This work can be extended in several directions. First, a mixed system consisting of automated and manual vehicles can be considered. Second, it would be useful to consider the urgency of a vehicle that wishes to change lane in order to further minimize the disruption of traffic flow. For instance, a vehicle needing to take an exit should be given a higher priority. Third, accelerations during lane changes may be explored to further increase the number of lane changes. Finally, while it is helpful to maximize the number of lane changes to alleviate its disruptive effects on traffic flow, the problem of deciding whether an automated vehicle should change lane in order to maximize throughput needs to be studied. 


\section{References}

[1] K. Ahmed, "Modeling drivers' acceleration and lane changing behavior," Ph.D. dissertation, Massachusetts Institute of Technology, 1999.

[2] T. Cowen, "Can I see your license and registration?" http://www.nytimes.com/2011/ 05/29/business/economy/29view.html

[3] B. Sen, J. Smith, and W. Najm, "Analysis of lane change crashes," National Highway Traffic Safety Administration, Technical Report, 2003.

[4] M. Roelofsen, J. Bie, L. Jin, and B. V. Arem, "Assessment of safety levels and an innovative design for the lane change assistant," in Intelligent Vehicles Symposium (IV), Oct. 1982 .

[5] H.-S. Tsao, R. Hall, and B. Hongola, "Capacity of automated highway systems: effect of platooning and barrier," California Partners for Advanced Transitand Highways (PATH), Technical Report, 1994.

[6] S. Habenicht, H. Winner, S. Bone, F. Sasse, and P. Korzenietz, "A maneuver-based lane change assistance system," in Intelligent Vehicles Symposium (IV), June 2011.

[7] G. Xu, L. Liu, Y. Ou, and Z. Song, "Dynamic modeling of driver control strategy of lane-change behavior and trajectory planning for collision prediction," Intelligent Transportation Systems, vol. 13, no. 3, pp. 1138-1155, 2012.

[8] J. Bascunana, "Analysis of lane change crash avoidance," in Future transportation technology conference and exposition, 1995.

[9] S. Hetrick, "Examination of driver lane change behavior and the potential effectiveness of warning onset rules for lane change or side crash avoidance systems," Master's thesis, Virginia Polytechnic Institute and State University, 1997.

[10] T. Wakasugi, "A study on warning timing for lane change decision aid systems based on driver's lane change maneuver," in Proceedings of the International Technical Conference on the Enhanced Safety of Vehicles, 2005.

[11] J. Li-sheng, F. Wen-ping, Z. Ying-nan, Y. Shuang-bin, and H. Hai-jing, "Research on safety lane change model of driver assistant system on highway," in Intelligent Vehicles Symposium, 2009.

[12] A. Amditis, M. Bimpas, G. Thomaidis, M. Tsogas, M. Netto, S. Mammar, A. Beutner, N. Möhler, T. Wirthgen, S. Zipser, A. Etemad, M. D. Lio, and R. Cicilloni, "A situation-adaptive lane-keeping support system: Overview of the safelane approach," Intelligent Transportation Systems, vol. 11, no. 3, pp. 617-629, 2010.

[13] M. Tomizuka, "Vehicle lane change maneuver in automated highway systems," California PATH Research Report, Technical Report, 1994. 
[14] W.Chee, "Unified lateral motion control of vehicles for lane change maneuvers in automated highway systems," California PATH Research Report, Technical Report, 1997.

[15] F. Wang, M. Yang, and R. Yang, "Conflict-probability-estimation-based overtaking for intelligent vehicles," Intelligent Transportation Systems, vol. 10, no. 2, pp. 366-370, 2009 .

[16] J. Naranjo, C. Gonzalez, R. Garcia, and T. de Pedro, "Lane-change fuzzy control in autonomous vehicles for the overtaking maneuver," Intelligent Transportation Systems, vol. 9, no. 3, pp. 438-450, 2008.

[17] J. Lygeros, D. Godbole, and S. Sastry, "Verified hybrid controllers for automated vehicles," Institute of Electrical and Electronic Engineers Transactions on Automatic Control, vol. 43, no. 4, pp. 522-539, Apr. 1998.

[18] R. Rajamani, H.-S. Tan, B. Law, and W.-B. Zhang, "Demonstration of integrated longitudinal and lateral control for the operation of automated vehicles in platoons," Control System Technology, vol. 8, no. 4, pp. 695-708, 2000.

[19] C. Hatipoglu, U. Ozüner, and K. Redmill, "Automated lane change controller design," Intelligent Transportation Systems, vol. 4, no. 1, pp. 13-22, Mar. 2003.

[20] I. Papadimitriou and M. Tomizuka, "Image compression using non-adaptive spatial vector quantization," in American Control Conference (ACC), June 2003.

[21] L. Wan, P. Raksincharoensak, and M. Nagai, "Study on automatic driving system for highway lane change maneuver using driving simulator," Journal of Mechanical Systems for Transportation and Logistics, vol. 4, no. 2, pp. 65-78, 2011.

[22] R. Schubert, K. Schulze, and G. Wanielik, "Situation assessment for automatic lanechange maneuvers," Intelligent Transportation Systems, vol. 11, no. 3, pp. 607-616, 2010.

[23] W. He, X. Wang, G. Chen, M. Guo, T. Zhang, P. Han, and R. Zhang, "Monocular based lane-change on scaled-down autonomous vehicles," in Intelligent Vehicles Symposium, 2011.

[24] R. Tomar and S. Verma, "Safety of lane change maneuver through a priori prediction of trajectory using neural networks," Network Protocols and Algorithms, vol. 4, no. 1, May 2012.

[25] R. Schubert and G. Wanielik, "Empirical evaluation of a unified bayesian object and situation assessment approach for lane change assistance," in Intelligent Transportation Systems, Oct. 2011.

[26] H. Jula, E. Kosmatopoulos, and P. Ioannou, "Collision avoidance analysis for lane changing and merging," Vehicular Technology, vol. 49, no. 6, pp. 2295-2308, Nov. 2000 .

[27] Y. Xuan and B. Coifman, "Lane change maneuver detection from probe vehicle dgps data," in Intelligent Transportation Systems, 2006. 
[28] P. Angkititrakul, R. Terashima, and T. Wakita, "On the use of stochastic driver behavior model in lane departure warning," Intelligent Transportation Systems, vol. 12, no. 1, pp. 174-183, Mar. 2011.

[29] S. Ammoun, F. Nashashibi, and C. Laurgeau, "An analysis of the lane changing manoeuvre on roads: the contribution of inter-vehicle cooperation via communication," in Intelligent Vehicles Symposium, 2007.

[30] L. Li, F.-Y. Wang, and Y. Zhang, "Cooperative driving at lane closures," in Intelligent Vehicles Symposium (IV), 2007.

[31] H. Park and B. Smith, "Investigating benefits of intellidrive in freeway operations: Lane changing advisory case study," Journal of Technology Education, vol. 138, no. 9, pp. 1113-1122, 2012.

[32] A. Bohm, M. Jonsson, and E. Uhlemann, "Adaptive cooperative awareness messaging for enhanced overtaking assistance on rural roads," in Proceedings of the Vehicular Technology Conference, 2011.

[33] P. Lytrivis, G. Thomaidis, M. Tsogas, and A. Amditis, "An advanced cooperative path prediction algorithm for safety applications in vehicular networks," Intelligent Transportation Systems, vol. 12, no. 3, pp. 669-679, 2011.

[34] M. Ardelt, C. Coester, and N. Kaempchen, "Highly automated driving on freeways in real traffic using a probabilistic framework," Intelligent Transportation Systems, vol. 13, no. 4, pp. 1576-1585, Dec. 2012.

[35] R. Horowitz and X. S. C.-W. Tan, "An efficient lane change maneuver for platoons of vehicles in an automated highway system," California PATH Research Report, Technical Report, 2004.

[36] H.-H. Hsu and A. Liu, "Platoon lane change maneuvers for automated highway systems," in Proceedings of the Conference on Robotics, Automation and Mechatronics, Dec. 2004.

[37] H. Hsu and A. Liu, "Kinematic design for platoon-lane-change maneuvers," Intelligent Transportation Systems, vol. 9, no. 1, pp. 185-190, Jan. 2008.

[38] M. Hilscher, S. Linker, E.-R. Olderog, and A. Ravn, "An abstract model for proving safety of multi-lane traffic manoeuvres," in Proceedings of the International Conference on Formal methods and Software Engineering, Oct. 2011.

[39] R. Hall and C. Caliskan, "Design and evaluation of an automated highway system with optimized lane assignment," Thomson Reuters Business Classification, vol. 7, no. 1, pp. 1-15, Feb. 1997.

[40] R. Hall and C. Chinan, "Vehicle sorting for platoon formation: Impacts on highway entry and throughput," Thomson Reuters Business Classification, vol. 13, no. 5, pp. 405-420, Oct. 2005. 
[41] D. Ramaswamy, J. Medanic, W. Perkins, and R. Benekohal, "Lane assignment on automated highway systems," Vehicular Technology, vol. 46, no. 3, pp. 755-769, Aug. 1997.

[42] K. Kim, D.-I. Cho, and J. Medanic, "Lane assignment using a genetic algorithm in the automated highway systems," in Intelligent Transportation Systems (ITS), 2005.

[43] P. Gipps, "A behavioural car-following model for computer simulation," Transportation Research Part B: Methodological, vol. 15, no. 2, pp. 105-111, Apr. 1981.

[44] A. Bohm and M. Jonsson, "Supporting real-time data traffic in safety-critical vehicleto-infrastructure communication," in Proceedings of the Conference Local Computer Networks, Oct. 2008.

[45] J. Santa, A. Gómez-Skarmeta, and M. Sánchez-Artigas, "Architecture and evaluation of a unified V2V and V2I communication system based on cellular networks," Computer Communications, vol. 31, no. 12, pp. 2850-2861, 2008.

[46] J. Neades and R. Ward, "Swerves and lane changes," Professional Development in Road Accident Investigation Course. http://www.mathtech.co.uk/Downloads/ Swerves $\backslash \% 20$ and $\backslash \% 20$ Lane $\backslash \% 20$ Changes.pdf 